\title{
Mechanisms of Terahertz Generation under Femtosecond Pulses propagation in Nanocomposites
}

\author{
O. Fedotova ${ }^{1}$, A. Husakou ${ }^{2}$, G. Rusetsky ${ }^{1}$, O. Khasanov ${ }^{1}$, \\ A. Fedotov ${ }^{3}$, T. Smirnova ${ }^{4}$, U. Sapaev ${ }^{5}$, I. Babushkin ${ }^{6,7,2}$ \\ 1. Scientific-Practical Materials Research Centre NAS Belarus, P.Brouki 19, Minsk, Belarus \\ 2. Max Born Institute, Max Born Str. 2a, 12489 Berlin, Germany \\ 3. Belarusian State University, Niezaliezhnasci avenue 4, 220030 Minsk, Belarus \\ 4. International Sakharov Environmental Institute BSU, Dauhabrodskaya str. 23, Minsk, Belarus \\ 5. Tashkent State Technical University, st. University 2, Olmazar district, 100095 Tashkent, Uzbekistan \\ 6. Institute of Quantum Optics, Leibnitz Hannover University, Welfengarten 1, 30167 Hannover, Germany \\ 7. Cluster of Excellence PhoenixD, Welfengarten 1, 30167 Hannover, Germany
}

Terahertz (THz) generation has a high potential for study vibrational and rotational transitions, dynamics of free carriers and phonon resonances, as well as applications for lasing, security systems, spectroscopy, biomedicine, ultrafast switching and controlling of magnetic domains, molecular alignment, particle acceleration, THz-enhanced attosecond pulse generation [1]. The challenge is to produce a compact intense THz- source, for that a development of schemes and devices providing high efficiency of frequency conversion is important. For the moment, it may reach a few percent in different schemes such as optical rectification, laser-solid interaction, two-color gas-plasma scheme, laser pumped liquid-plasma [2]. Prospective media for these aims are nanocomposites (NC) due to their large nonlinearities bringing wide set of the possibilities. Large value of permanent dipole moment (PDM) is revealed $\left(\sim 10^{2}-10^{3}\right.$ Debye) in nanostructures, in particular, semiconductor quantum dots (QDs) of $\mathrm{ZnO}, \mathrm{ZnS}, \mathrm{CdSe}$ what is comparable with values of transition dipole moments between the exciton states. As well, the additional transitions between exciton states allowed due to PDM may play a significant role in the nanoparticle response, in particular for the generation of new frequencies in the $\mathrm{THz}$ range.

We study $\mathrm{THz}$ generation in NC consisted of QD inclusions $(\mathrm{ZnO})$ in transparent dielectric matrix $\left(\mathrm{SiO}_{2}\right.$ host). Pumping pulse carrier frequency is supposed to be in resonance with a transition between lower excitonic levels. Theoretical model allowing to study coupled resonant and non-resonant mechanisms of down frequency conversion in nanocomposite has been developed. Simulations of the pulse propagation were performed on the base of self-consisted system for the density matrix (Bloch) equations describing multilevel excitonic transitions and the unidirectional propagation equation, accounting for chromatic dispersion, second- and third-order optical nonlinearities of both host and inclusions, photoionization of inclusions, plasma dynamics and its influence on the dielectric function of the inclusions. Numerical simulations were performed using a SOLPIC software developed in the frame of RISE-ATLANTIC project. As an analysis shows, for the case of smaller PDM and input intensity $0.055 \mathrm{TW} / \mathrm{cm}^{2}$ the output efficiency of $\mathrm{THz}$ may reach $0.23 \%$ after $50 \mu \mathrm{m}$ of propagation of two 15 -fs pulses at central circular frequencies of $2.26 \mathrm{fs}^{-1}$ and $2.40 \mathrm{fs}^{-1}$, what may be explained by significant exciton resonance contribution. In Fig.1 (a,b) the results are shown for 15 -fs-pulse with an input intensity $1 \mathrm{TW} / \mathrm{cm}^{2}$ and central frequency $\omega_{0}=2.26 \mathrm{fs}^{-1}$ (FWHM) propagating over distances 10 and $50 \mu \mathrm{m}$ in NC with 100 Debay PDM. It is established that the $\mathrm{THz}$ part of the spectrum is increased with the propagation distance. For comparison, the model case without PDM but the same other parameters is presented in Fig.1 (c), from this one can conclude that $\mathrm{THz}$ efficiency at the same propagation length is by 2-3 order of magnitude more for the case with PDM.

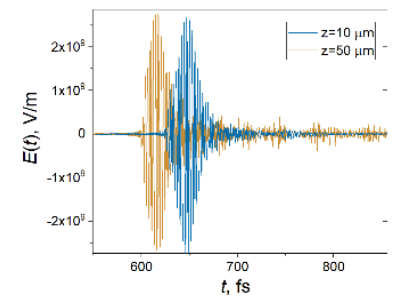

(a)

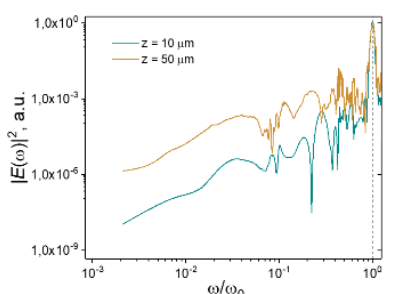

(b)

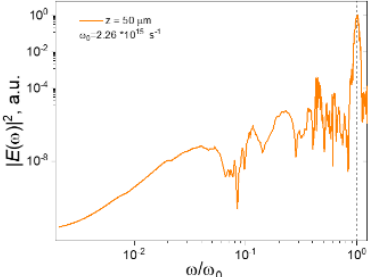

(c)

Fig. 1 Temporal pulse profiles (a) and spectra (b,c) of the laser pulse propagating in nanocomposite over 10 and 50 $\mu \mathrm{m}$ for parameters given in text with PDM d=100 Debay (b) and without PDM (c).

\section{References}

[1] A.D. Koulouklidis, C. Gollner, V. Shumakova, Vladimir Yu. Fedorov, Audrius Pugžlys, Andrius Baltuška and Stelios Tzortzakis, "Observation of extremely efficient terahertz generation from mid-infrared two-color laser filaments", Nat Commun 11, 292 (2020). [2] S. B. Bodrov, A. N. Stepanov, and M. I. Bakunov, "Generalized analysis of terahertz generation by tilted-pulse-front excitation in a $\mathrm{LiNbO}_{3}$ prism," Opt. Express 27, 2396-2410 (2019). 